\title{
Évaluation de degré de défense de l'architecture défensive pré- coloniale en Algérie : cas des villages fortifiés
}

Assassing the defensibility of the pre-colonial defensive architecture in Algeria: case study on the medieval fortified villages

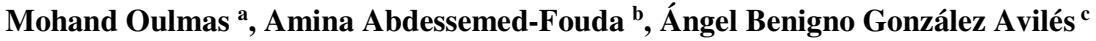 \\ ${ }^{a}$ Laboratoire Environnement et Technologie pour l'Architecture et le Patrimoine - Université Saâd Dahleb, Blida 1, \\ Algeria, oulmas.mohand15@gmail.com \\ b Laboratoire Environnement et Technologie pour l'Architecture et le Patrimoine - Université Saâd Dahleb, Blida 1, \\ Algeria, aafoufa@univ-blida.dz \\ ${ }^{\mathrm{c}}$ Departamento de Construcciones Arquitectónicas - Universidad de Alicante, Alicante, Spain, angelb@ua.es
}

\begin{abstract}
Algeria's pre-colonial towns of the medieval period still exist in different typologies, ranging from the isolated buildings (forts, castles) and town enclosures to whole urban units (fortified villages, defensives towns). Indeed, the constituent of these fortresses was their defense system, characterized by its large dimension, constituted essentially by the enclosure wall, and architectural features of defensiveness correlated with the outside and the inside of the fortresses. This paper aims to evaluate the relationship between physical landscape, built defensive features and cultural values of the medieval fortified villages in Algeria, two medieval fortified villages in our case "Kalaa of Beni Abbes" in Bejaia and "Kalaa of Beni Rached" in Oran, that we identified as an evolved landscape and interpreted as complex system (both defensive architecture and continuing cultural landscape). This current study consists of quantifying the defensiveness degree of these sites situated within different contexts, in fact, this method ensures to identify the strategy adopted to be protected against different invasions. However, in order to achieve this we calculate a spatial defensiveness index (DI) of these sites. The parameters of our choice are related to the implantation site, the elevation, the visibility and the geometrical shape, which allow us to estimate the defensiveness degree of the defense system of our case studies.
\end{abstract}

Keywords: Defensibility, medieval villages, defensive architecture, Algeria.

\section{Introduction}

Les diverses formes de l'architecture défensive de la période médiévale désigné par le mot kalaa dans son acception la plus usuelle, ont toujours constitué l'élément principal des fortifications qui se sont élevées en territoire de l'Islam, dès les premières années de la conquête musulmane des territoires occidentaux du monde islamique, lors de l'invasion des musulmans à partir de 647 de l'an J.C. de l'Ifriqiya (Tunisie), Le Maghreb, le Maghreb central (Algérie) et l'Espagne. En l'an 670 sous le commandement d'Okba Ibn Nafia à la fin de laquelle le Maghreb est devenu une province musulmane avec Kairouan comme capitale, cette conquête a duré environ 70 ans, accompagnée par l'établissement des tribus et bases de chacune des provinces conquises par les différentes dynasties musulmanes. Ces $k a$ laas étaient autrefois des symboles de pouvoir et d'autorité, érigées autour des préoccupations défensives et stratégiques qui mettent à profit leur 
implantation. Ces dernières subsistent sous différentes formes et typologies, parfois en ouvrage isolé, en enceinte de villes allant jusqu'en unité urbaine. Il ressort aujourd'hui que certaines d'entre elles se trouvant au cœur même des villes dont elles marquent des territoires, des paysages contribuant à leur identité.

Cette présente étude a pour but de trouver la relation entre le paysage naturel, les éléments de défense de ces forteresses en évaluant leur degré de défense, qui nous permettrait de comprendre l'évolution de leur système défensif. En effet, afin de mettre en pratique ce travail, nous nous sommes appuyés sur la méthode élaborée par Andrew Martindale et Kisha supernant (2009), appliquée sur les fortifications médiévales dans un contexte canadien, en estimant le coefficient de défense du paysage classé fortifié, représenté par un indice de défense (DI : "defensiveness inde", dérivé de 4 variables distinctives : la visibilité, l'élévation, l'accessibilité et la surface. Pour notre cas nous testons cette approche afin d'identifier et évaluer la stratégie défensive adoptée par le constructeur médiéval que nous appliquerons sur deux cas de figues: la forteresse de Béni Abbès à Bejaia, la forteresse de Béni Rached à Relizane, deux fortifications de typologie village fortifié, conçues durant la période médiévale et qui représentent des similitudes sur le plan défensif, situées dans deux contextes différents. En revanche, cela va nous conduire à une étude comparative entre les indices de défense pour chaque site, de manière à définir leur stratégie défensive adoptée, et mettre en évidence les critères d'implantation, le choix d'angle de vision, la distance par rapport à la mer et la montagne, les caractéristiques architecturales ainsi que les voies de communication, qui sont tous des facteurs que nous avons jugés important dans notre étude.

\subsection{Approche méthodologique}

L'étude des différents traits paysagers des forteresses étudiées pourrait constituer des formes de défense similaires. A partir d'une étude comparative nous essayerons d'estimer la cohérence défensive, par ailleurs, notre démarche est double, premièrement nous allons rechercher les mesures de défense à caractère naturel (implantation), et deuxièmement des caractéristiques architecturales et paysagères qui créent souvent des arrangements avec le contexte naturel pour une meilleure protection contre l'ennemi, en d'autres termes, nous recherchons à justifier les préoccupations défensives du constructeur médiéval, et cela à partir d'une approche quantifiable.

\subsection{Calcul de l'indice de défense selon la mé- thode Andrew Martinandale et Kisha Super- nant}

Dans leur recherche, Martinandale et Supernant (2009), considèrent que pour calculer un degré de défense d'un site classé fortifié, il suffit de calculer son indice de défense [DI], qui luimême est représenté par une valeur unique, dérivé de 4 mesures distinctives: la visibilité, l'élévation, l'accessibilité du site et sa surface. Nous appliquerons dans notre recherche cette approche sur un échantillon de plusieurs sites, néanmoins dans ce présent travail nous limitons notre choix à la typologie : fortification-village "la Kalaa de Béni Abbès à Béjaia et la Kalaa de Béni Rached à Relizane", chacune dans son contexte d'appartenance. La capacité défensive sera calculée en tenant en compte le critère d'implantation, les caractéristiques architecturales et paysagères. Cet indice permet d'estimer la défense d'un système spatial entre l'intérieur et l'extérieur et varie entre 0 et 4 , dont sa valeur représente la somme des 4 facteurs choisis : la visibilité, l'élévation, l'accessibilité et la surface du site.

Dans cette recherche Notre objectif est de justifier le paramètre conduisant à un tel système défensif, et arriver à comprendre le système défensif des villages défensifs de la période médiévale en Algérie, et désigner sa cohérence, qu'il soit relatif au choix d'implantation ( donc visibilité et dénivelé), aux éléments architecturaux défensif, à la forme géométrique, l'accessibilité ou à la surface, en terme simple qu'est ce qui permet de dire que le degré de défense d'une forteresse est plus élevé qu'une autre.

Afin de quantifier cet indice de défense, elles sont prises deux variables extrêmes : 
Une défense nulle qui représente un plan sans barrière et protection : la valeur défensive $=0$.

Et une défense maximale qui représente un espace protégé et inaccessible avec une valeur défensive $=4$.

Nous notons que les résultats obtenus sont calculés à partir d'une méthode mathématique en utilisant des preuves empiriques et documents graphiques.

\section{Méthode de calcul}

Les composantes de l'indice de défense sont définies comme suit :

\section{La visibilité (V)}

Selon Andrew Martinandale et Kisha Supernant La visibilité se mesure comme un champ de vision, qui comprend à la fois l'arc de vision et la distance dans le champ de vision. Ainsi, chaque degré de direction autour d'un site pourrait être calculé en fonction de la valeur de la distance, les distances plus courtes représentant moins de défense. Un moyen alternatif et plus simple de se rapprocher de la défense consiste à supposer que la visibilité minimale est fonctionnellement limitée à $100 \mathrm{~m}$ (à partir de l'enceinte de la forteresse au positionnement de l'ennemi).

La visibilité $(v)=$ degré de visibilité à une distance supérieure à $100 \mathrm{~m} /$ le degré d'approche autour du site.

La visibilité obtenue par rapport à la mer ou au terrain, se divise sur un degré approche, qui est représenté par un arc total de $360^{\circ}$, cela permet de normaliser les résultats obtenus dans une plage qui varie entre 0 et 1 (Martinandale, Supernant, 2009).

\section{L'élévation (E)}

L'élévation peut être exprimée par des différences d'altitude ou de pente du point le plus élevé d'un site. Nous utilisons une mesure radiale des différences d'altitude en degrés au lieu de la pente $[(\mathrm{m})=\mathrm{ev} / \mathrm{d}]$ pour éviter une fonction logarithmique. Conversion des degrés d'élévation en une fraction de 90 convertis, le calcul est compris entre 0 et 1 (Martinandale, Supernant, 2009).

Elévation $(E)=E v($ degré de l'élévation $) / 90^{\circ}$.

\section{L'accessibilité (C)}

L'accessibilité du site : définit dans notre cas, comme le degré de protection de la forteresse, c'est-à-dire avoir un pourcentage des obstacles qu'ils soient bâtis (éléments de défense construits) ou bien naturels : relief, arbres ... qui dans leur globalité rendent la forteresse fortement inaccessible ou l'inverse, selon le contexte de sa construction (Martinandale, Supernant, 2009).

Accessibilité $(c)=[360-P$ (degré d'approche autour $d u$ site)/360] $+[P-T$ (degré d'accessibilité par rapport aux seuils de la forteresse)/p]

\section{La surface (S)}

La surface pourrait être un facteur déterminant de degré de défense d'un site fortifié, plus la surface du site est importante, plus on dit que le site est difficile à attaquer. Pour avoir un ratio d'accessibilité à un site, nous allons diviser la surface du site sur une surface maximum que nous avons défini à $1000000 \mathrm{~m}^{2}$ ((Martinandale, Supernant, 2009).

$S=$ surface du site $/ 1000000$

\section{Application aux cas d'étude.}

\section{1. Étude l'évolution du système défensif des forteresses de la période médiévale en Algérie de la conquête arabe à l'occupation espagnole}

Pour une plus grande clarification sur la question des origines de l'évolution du système défensif en Algérie durant la période, nous avons choisi de les étudier dans leur chronologie historique et dans leur contexte géographique.

En Algérie, au début de la conquête, les musulmans se contentèrent d'occuper certaines places fortes et citadelles antiques, notamment byzantines. Ce n'est qu'à partir du VIII ${ }^{\text {ème }}$ siècle de l'ère chrétienne que les arabes commencèrent à fonder leurs propres fortifications, souvent inspirés d'autres répertoires architecturaux, notamment celui de l'antiquité, que nous avons par conséquent répartis en trois catégories : 
- Les fortifications érigées entre le VIII $I^{\text {ème }}$ et $X^{\text {ème }}$ siècle

- Celles construites entre le XI $I^{\text {ème }}$ et XII ${ }^{\text {ème }}$ siècle

- D'autres qui ont été bâties entre le $X^{X I I}{ }^{\text {ème }}$ et début du XVI ${ }^{\text {ème }}$ siècle

Les fortifications érigées entre le VIII ${ }^{\text {ème }}$ et $\mathbf{X}^{\text {ème }}$ siècle : Première forme de fortifications de la période musulmane, tour (carrée ou ronde) attenante à un rempart ou bien isolée et servant de bastion ou de donjon. Le Burdj fait partie des dispositifs byzantins, il s'agit d'un modèle superposé sur une base antique : typologie de modèle superposé occupé par les premiers musulmans (Fig. 1).

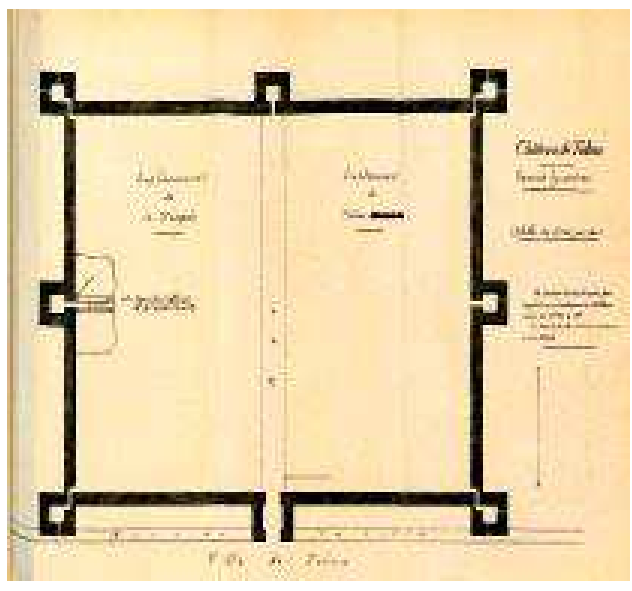

Fig. 1. Tobna, batna, Algérie. Transformation du plan par les musulmans (Bourouiba, 1983).

Celles construites entre le $\mathrm{XI}^{\text {Ìme }}$ et $\mathrm{XII}^{\text {ème }}$ siècle: Modèle inspiré de l'architecture du moyen orient : burdj isolé, base talutée, fournit une indication sur la nature du fossé qui entoure la forteresse, avec flanquements, et enceinte adaptée au contexte topographique (Fig. 2), sa Typologie architecturale se réduit à 4 éléments) :

1) Le dispositif de défense : inclus la muraille d'enceinte, parfois doublée d'une deuxième fortification, ou carrément en double rempart

2) Le noyau fondamental : inclus le centre religieux et culturel et un centre du système commercial et le centre politico-administratif où converge le réseau routier primaire.

3) Unité résidentielle
4) Une courtine à des hauteurs variable si elle existe

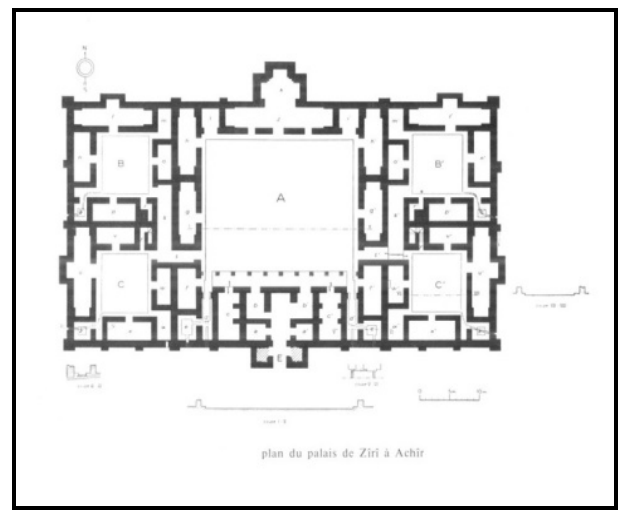

Fig. 2. Plan de la forteresse d'Achir (Encyclopédie berbère 1989).

D'autres qui ont été bâties entre le XIII $I^{\text {ème }}$ et début du XVI $I^{\text {ème }}$ siècle : Les Qalaas ont été fondées, car les villes ouvertes sont devenues rares dans le monde occidental, ainsi que les troubles dus aux invasions aient menés les centres urbains à se couvrir d'une muraille. L'enceinte est à un seul mur ou parfois à double, et comporte rarement de donjon ou de tours flanquantes, aux fondations des fatimides des nouveautés apparaissent, les murailles de leurs fortifications flanquées de tours puissantes, une d'entre elle était décorée des hautes niches qui ornèrent plus tard la Qalaa de Banu-Hammad (Fig. 3).

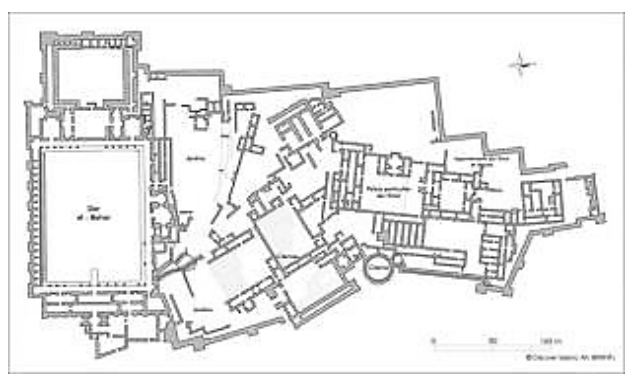

Fig. 3. Qalaa de banu-Hammad, régularité des formesPlan d'ensemble (Golvin, 1957).

Toutefois, dans le monde du Grand Maghreb, que ça soit en plaine ou en crête, les dynasties musulmanes amenaient à fortifier les villes ou à entretenir leurs murs, nous avons par ailleurs répertorié deux typologies de ces kalaas : les $k a$ laas inspirées des modèles orientaux (Fig. 3), et un modèle autochtone avec une architecture plu- 
tôt traditionnelles, typologie village fortifié (Fig. 4 ), et ce sont ces modèles les plus conservés.

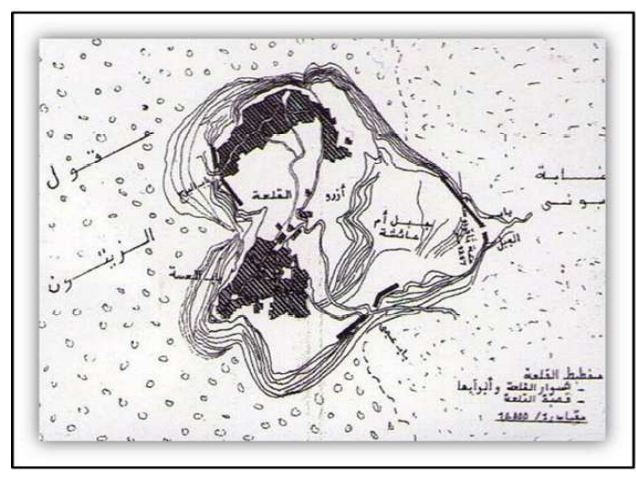

Fig. 4. Implantation de la Kalaa de Beni Abbès, Kalaa de typologie village fortifié (http://www.gehimab.org).

Un autre type de forteresse fut apparu durant l'occupation espagnol au milieu du XVI ${ }^{\text {ème }}$ siècle, à l'exemple du fort Burdj Moussa (Fig. 5), une typologie d'étoile, édifié par les espagnols. Le plan est dessiné par Librano, avec une architecture plus régulière, d'une forme rectangulaire avec des bastions latéraux, qui contient une enceinte à talus dont la hauteur est de 100 pieds ayant des murs inclinés. En effet, ce système défensif gigantesque est généralement adopté dans les constructions en plaine, afin d'avoir un degré de défense important.

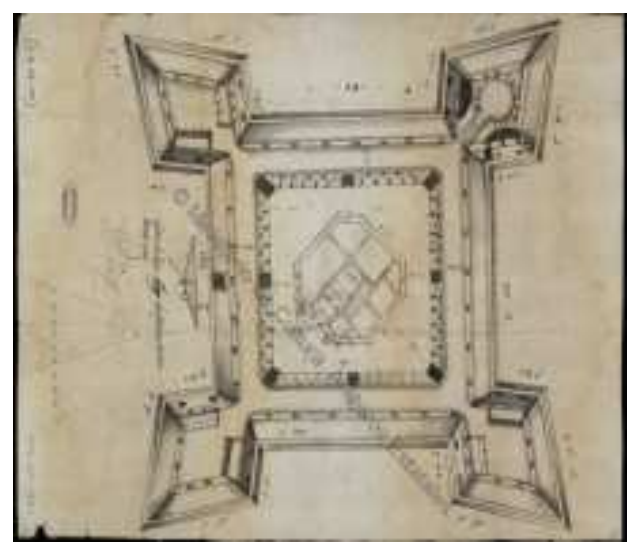

Fig. 5. Plan de Burdj Moussa, Bejaia, Algérie (les archives de Simancas).
3.2. Quantification de la défensibilité des villages fortifies en Algérie : cas de la Kalaa des Bénis Abbès à Béjaia, et la Kalaa des Béni Rached à Oran

Dans cette présente recherche nous nous focalisons sur deux forteresses la Kalaa de Béni Abbés à Béjaia et la forteresse de Bénis Rached à Relizane, ces deux villages fortifiés datent de la période médiévale, leur édification a connu trois moments de l'histoire distinctifs; la conquête arabe, l'occupation espagnol et la colonisation française. Ces deux villages représentent des caractéristiques architecturales similaires, mais un système défensif différent, en revanche, nous testons par cette rechercher évaluer leur degré de défense [DI], à partir d'une méthode quantifiable qui permet de comprendre la stratégie adoptée pour obtenir le système défensif pour chaque site.

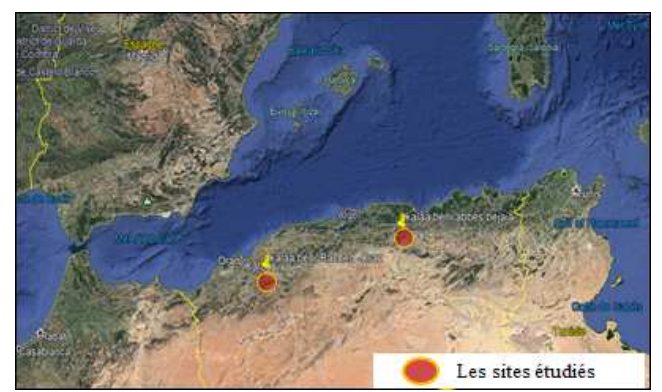

Fig. 6. Implantation des deux villages sur la côte méditerranéenne (Google Earth).

Premier cas : La Kalaa de Béni Abbès, à Béjaia, perchée sur un site très accidenté $(1010 \mathrm{~m}$ d'altitude), d'une enceinte mono, et partielle, forme architecturale irrégulière (Fig. 7).

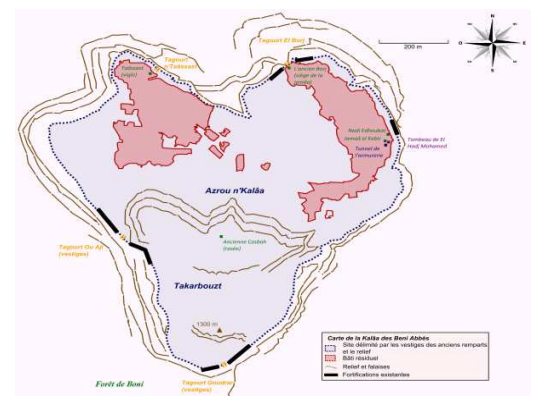

Fig. 7. La Kalaa de Béni Abbès à Béjaia (http://www.gehimab.org). 
Deuxième cas : la Kalaa de Béni Rached à Oran, implantée sur un site moyennement accidenté (477 m d'altitude), enceinte globale, renforcée par des murailles pour chaque unité qui compose la Kalaa, sans tours de surveillances (Fig. 8).

Le tableau suivant (Fig. 9) récapitule les résultats des indices de défense obtenu en utilisant la méthode mathématique en se basant sur des données graphiques et empiriques. La représentation graphique est illustrée dans les figures 10 et 11 .

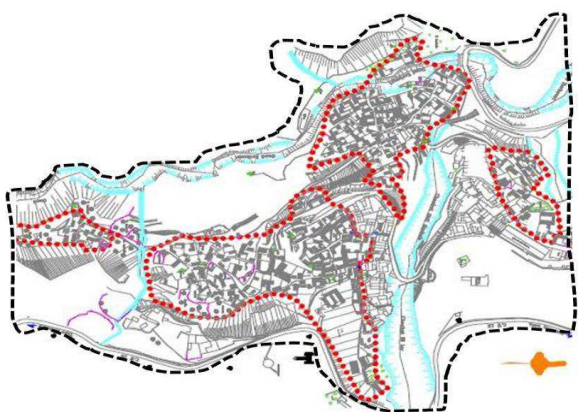

Fig. 8. La Kalaa de Béni Rached à Relizane, Oran (bureau d'étude URBA-BLIDA).

\begin{tabular}{|c|c|c|c|c|c|c|c|c|c|c|c|c|}
\hline $\begin{array}{l}\text { Nom du } \\
\text { site }\end{array}$ & Type & $\begin{array}{c}\text { Approach } \\
\text { degrees: } \\
\text { land }\end{array}$ & $\begin{array}{c}\text { Approach } \\
\text { degree : } \\
\text { water }\end{array}$ & $\begin{array}{c}\text { Visibility } \\
\text { in degrees } \\
\text { (land) } \\
\geq 100\end{array}$ & $\begin{array}{c}\text { Elevation } \\
\text { changes } \\
\text { (m) }\end{array}$ & $\begin{array}{c}\text { Degrees } \\
\text { access }\end{array}$ & Area m2 & $\begin{array}{c}\text { Visibility } \\
\text { (v) }\end{array}$ & $\begin{array}{c}\text { Elevation } \\
\text { (E) }\end{array}$ & $\begin{array}{l}\text { Approch } \\
\text { access (c) }\end{array}$ & $\begin{array}{c}\text { Area } \\
\text { (A) }\end{array}$ & $\begin{array}{c}\text { DI } \\
\text { (defensiveness } \\
\text { Index) } \\
\end{array}$ \\
\hline $\begin{array}{c}\text { 1. Kalaa } \\
\text { de béni } \\
\text { Abbès }\end{array}$ & $\begin{array}{l}\text { Village } \\
\text { fortifié }\end{array}$ & 180 & 180 & 190 & 54 & 190 & 845972.25 & 1 & 0,51 & 0,042 & 0,85 & 2,40 \\
\hline $\begin{array}{l}\text { 2. Kalaa } \\
\text { de Béni } \\
\text { Rached }\end{array}$ & $\begin{array}{l}\text { Village } \\
\text { fortifié }\end{array}$ & 180 & 0 & 50 & 40 & 270 & 107177,15 & 0,45 & 0,23 & 0,43 & 0,10 & 1,21 \\
\hline
\end{tabular}

Fig. 9. Tableau récapitulatif des résultats du calcul de l'indice de défense [DI], en fonction de la visibilité, l'élévation, accessibilité et la surface, selon la méthode de Martinandale et Supernant (2009).

\subsection{Discussion des résultats obtenus}
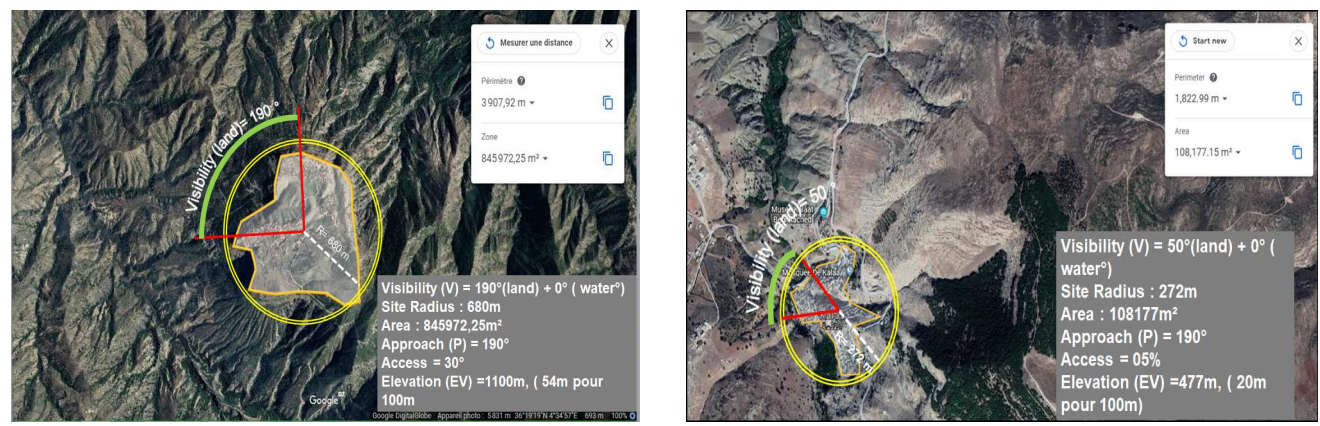

Figs. 10-11. Représentation graphique des données. Kalaa de Béni Abbès à Bejaia (gauche) et à Oran (droite).

Nous avons constaté que les premiers paramètres pris en considération par constructeur médiéval sont liés à l'implantation, qui est elle aussi directement liée aux facteur naturel. Ces facteurs peuvent être interprétés dans notre cas par la visibilité (1), et l'élévation (0,51), qui euxmêmes augmentent ou réduisent l'accessibilité au site et offre une meilleur protection contre l'enemi, comme nous pouvons remarqué dans le cas de la forteresse de Béni abbès à Bejaia, la visibilité est très importante, cela est permi grace à son implantation en relief accidenté qui luimême répond aux exigences défensives. En effet, ce village défensif, contient une rempart sur un seul côté (côté frontal), qui donne sur la mer, et toute la partie arrière, est déjà protégée par la montagne (Fig. 11). Pour ce cas de figure le facteur le plus important pour avoir le 
meilleur indice de défense est l'élévation (altitude). L'indice de défense [DI] est estimé à $2,40 / 4$, ce qui fait que la forteresse soit parfaitement protégée, et inaccessible.

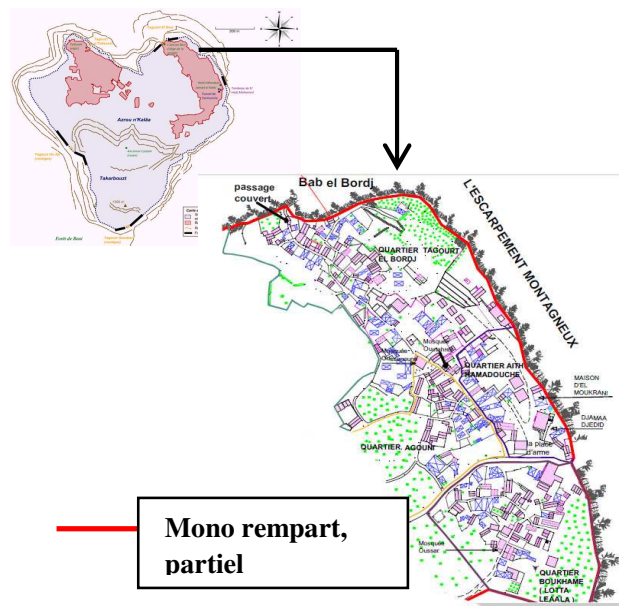

Fig. 12. Plan du Rempart unilateral, Kalaa de Béni Abbès, Bejaia, Algérie.

Pour le deuxième cas de figure, la Kalaa de Béni Rached, le facteur le plus important est l'inaccessibilité $(0,45)$. Cette dernière est obtenu grace à la pésence d'un rempart globale sur toute la forteresse, d'autre doublé sur chaque unité qui compose la Kalaa. En effet, cette stratégie a été adoptée car l'élévation n'est pas importante (447 m) comparativement au premier cas d'étude.

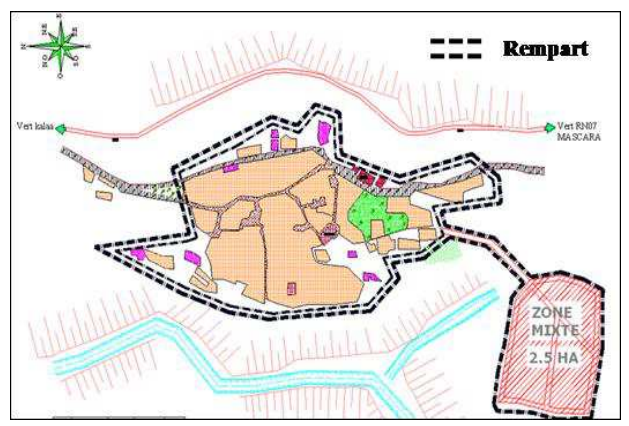

Fig. 13. Double rempart, Kalaa de Béni Rached, oran, Algérie.

Nous pouvons déjà à partir de cette comparaison repérer les paramètres pris en considération lors de la construction des villages médiévaux en Algérie. En effet, des conditions d'implantation et les facteurs naturels sont les éléments qui dictent la stratégie défensive adoptée dans chaque contexte, par ailleurs nous avons obtenu deux possibilités de défense :

Sur un site fortement accidenté, comme le cas de la Kalaa de Béni Abbès: la Kalaa est principalement protégé par son site, et cette implantation lui offre une visibilité de $100 \%$ sur les alentours (distance minimale 100m). le système défensif utilisé est économique, car une seule partie est protégée par un rempart, quant à la partie arrière est déjà naturellement protégé par la montagne. Contraiement à la Kalaa de Béni Rached à Oran, d'autres exigences défensives ont été adoptées, autre que l'élévation qui est basse, pour rattraper cela, le constructeur a renforcé tout le village par un rempart. Nous ajoutons, qu'au lieu de doubler la structure d'enceinte, et aussi pour des raisons économique, chaque unité qui compose la forteresse est renforcé par un autre rempart (Fig. 13).

\section{Conclusion}

Cette méthode d'évaluation du degré de défense des forteresses médiévales en Algérie, inspirée par la démarche canadienne développée par Martinandale et Supernant, nous a permi de comprendre la stratégie défensive employée par le constructeur de la période médiévale.Pour le cas des villages défensifs étudiés, nous avons conclu que l'élévation et la visibilité sont deux facteurs principaux déterminants de l'indice de défense [ DI] le plus élevés: plus l'élévation est importante, plus le l'indice de défense est important. Dans un autre cas, où l'implantation à une altitude basse ou moyenne, comme l'exemple de la Kalaa de Béni Rached à Relizane, le facteur déterminant est l'accessiblité, c'est-à-dire: construire des remparts à des hauteurs importantes pour rendre la forteresse inaccessible et protégee.

Nous sommes enfin arrivés à déduire que les facteurs naturels (l'implantation) reste une condition primordiale et décisive pour déterminer un degré de défense important dans les sites classés fortifiés. 


\section{Bibliography}

Bourouiba, R. (1983). L'architecture militaire de l'Algérie médiévale, Algérie, office des publications universitaires Amman, pp. 12-50.

Cuneo, P. (1998). Introduction à l'urbanisme en pays de l'Islam et l'histoire des villes du Maghreb au moyen orient. Rome, Centre Analisi Sociale Progretti, SRL Ed., Paris, pp. 25-75.

Hecham Zeioua, B. (2014). "Biens culturels algériens et leur inscription sur la liste du patrimoine mondial : pour quels démarche et critères de sélection?", Laturbe: Revista euromediterránea de ciencias de la ciudad, del territorio y del medio ambiente, 2 (online).

Kyle Bocinsky, R. (2014). "Extrinsic site defensibility and landscape-based archaeological inference: an example from the Northwest Coast", Journal of Anthropological Archaeology, 35, pp. 164-176.

Marçais, G. (1958). Algérie médiévale, monuments et paysage historique, Art et métiers Ed., Paris, pp. 67.116.

Martindale, A.; Superrnant, K. (2009). "Quantifiying the defensiveness of defended sites on the Northwest Coast of North America”, Journal of Anthropological Archaeology, 28(2), pp. 191-204.

Sari, D. (1970). Les villes précoloniales de l'Algérie occidentale : Nedroma, Mazouna, Kalaa, Société nationale d'édition et de diffusion Ed., Alger, pp. 12-84.

Voisin, J.C. (2013). L'architecture militaire de l'Algérie: de Byzance à l'arrivée des Beni Hillal (VIe-XIIe siècle), Edition Barzakh, Alger, pp. 26-69. 